\title{
Global Functional Connectivity Deficits in Schizophrenia Depend on Behavioral State
}

\author{
Roy Salomon, ${ }^{1,2 \star}$ Maya Bleich-Cohen, ${ }^{2,3 *}$ Avital Hahamy-Dubossarsky, ${ }^{4 \star}$ Ilan Dinstien, ${ }^{4}$ Ronit Weizman, ${ }^{3,6}$ \\ Michael Poyurovsky, ${ }^{5}$ Marina Kupchik, ${ }^{7}$ Moshe Kotler, ${ }^{2,7}$ Talma Hendler, ${ }^{1,2,3}$ and Rafael Malach ${ }^{4}$ \\ ${ }^{1}$ Functional Brain Imaging Center, Wohl Institute for Advanced Imaging, Sourasky Medical Center, Tel Aviv 64239, Israel, ${ }^{2}$ Psychology Department and \\ ${ }^{3}$ Sackler Faculty of Medicine, Tel Aviv University, Tel Aviv 69978, Israel, ${ }^{4}$ Department of Neurobiology, Weizmann Institute of Science, Rehovot 76100, \\ Israel, ${ }^{5}$ Research Unit, Tirat Carmel Mental Health Center, Tirat Carmel 30200, Israel, ${ }^{6}$ Tel Aviv Community Mental Health Center, Tel Aviv 67197 Israel, \\ ${ }^{7}$ Beer Yaakov Mental Health Center, Beer Yaakov 70350, Israel
}

Schizophrenia is a devastating psychiatric illness characterized by deterioration of cognitive and emotional processing. It has been hypothesized that aberrant cortical connectivity is implicated in the disease (Friston, 1998), yet previous studies of functional connectivity (FC) in schizophrenia have shown mixed results (Garrity et al., 2007; Jafri et al., 2008; Lynall et al., 2010). We measured FC using fMRI in human schizophrenia patients and healthy controls during two different tasks and a rest condition, and constructed a voxelbased global FC index. We found a striking FC decrease in patients compared with controls. In the task conditions, relatively weaker FC was specific to regions of cortex not active during the task. In the rest condition, the FC difference between patients and controls was larger and allowed a case-by-case separation between individuals of the two groups. The results suggest that the relative reduction of FC in schizophrenia is dependent on the state of cortical activity, with voxels not activated by the task showing higher levels of FC deficiency. This novel finding may shed light on previous reports of FC in schizophrenia. Whether this neural characteristic is related to the development of the disorder remains to be established.

\section{Introduction}

Schizophrenia is a devastating mental disorder affecting $\sim 1 \%$ of the population worldwide (Bhugra, 2005). It has been hypothesized that schizophrenia is linked to a breakdown in cortical connectivity, which is measured by assessing the correlation between neural activity in different brain regions (Friston et al., 1993). The disconnection hypothesis proposes that reduced connectivity between different brain regions underlies impairments found in schizophrenia (Friston and Frith, 1995).

However, imaging studies of functional connectivity (FC) in schizophrenia have yielded mixed results (McGuire and Frith, 1996; Meyer-Lindenberg et al., 2001; Lawrie et al., 2002; Honey et al., 2005; Jafri et al., 2008; Liu et al., 2010). Several studies have reported reduced FC in schizophrenia. For example, reduced correlations between different cortical regions during an $n$-back memory task (Meyer-Lindenberg et al., 2001) and in a faceprocessing task (Bleich-Cohen et al., 2009) have been shown.

Received June 14, 2011; accepted July 11, 2011.

Author contributions: R.S., M.B-C., R.W., M.P., M. Kupshik, M. Kotler, and T.H. designed research; R.S., M.B-C., R.W., M. Kupshik, T.H., and R.M. performed research; R.S., M.B-C., A.H-D., I.D., T.H., and R.M. analyzed data; R.S., M.B-C., A.H-D., I.D., T.H., and R.M. wrote the paper.

This study was supported by the Israel Science Foundation (ISF 160/07), EU-VERE-FT grant, and The Hellen Kimmel Award for Innovative Research to R.M. We thank Y. Nir, G. Gilam, G. Hesselmann, M. Ramot, Adams Super Center for Brain Studies, Tel Aviv Medical Center, the patients, and all lab members for their help in various stages of this project.

*R.S., M.B-C., and A.H.D. contributed equally to this research.

The authors declare no conflict of interest.

Correspondence should be addressed to Rafael Malach at the above address. E-mail: rafi.malach@gmail.com. DOI:10.1523/JNEUROSCI.2987-11.2011

Copyright $\odot 2011$ the authors $\quad 0270-6474 / 11 / 3112972-10 \$ 15.00 / 0$
However, others have reported increased connectivity during cognitive tasks (Jafri et al., 2008; Whitfield-Gabrieli et al., 2009). For example, increased FC between regions of the default mode network (DMN) in both schizophrenia patients and healthy relatives during a working memory task have been found (Whitfield-Gabrieli et al., 2009). Thus, experimental findings have been inconsistent and vary depending on the specific paradigm.

Recently, resting-state fMRI (rsfMRI) has been used to study patients with brain pathologies whose ability to cooperate and perform tasks is disrupted. This method is suited to study pathologies as it is not confounded with task-specific activity patterns or performance differences between patients and healthy controls. Results from rsfMRI in schizophrenia have also revealed varied results, with some studies finding reduced FC in schizophrenic patients (Liang et al., 2006; Bluhm et al., 2007; Liu et al., 2008) while others have found increased FC (Zhou et al., 2007; Whitfield-Gabrieli et al., 2009; Salvador et al., 2010). Most of these studies used a seed-based approach (i.e., testing the connectivity of a predefined region within the brain), limiting analysis to specific regions. Other studies, which assess functional connectivity more widely throughout the brain, have reported reduced connectivity in schizophrenia (Liu et al., 2008; Lynall et al., 2010). Together, these studies show aberrant FC in schizophrenia with variable results as to the directionality of the anomalous connectivity.

Here we compared schizophrenia patients and healthy controls while they performed two cognitive tasks. Using a quantitative measure of connectivity for each voxel, we found a striking 
disruption of functional connectivity in the schizophrenia patients. Intriguingly, this relative disruption was state-dependent and localized to regions not activated by each task, suggesting that the FC impairment is related to the level of task-related activity in the cortex. To further test this hypothesis, we conducted a second experiment in a group of first-episode schizophrenia subjects using rsfMRI and found that the FC decrease in the schizophrenia patients was even larger during the rest condition. These results point to the activity state of the cortex as a critical parameter affecting the strength of FC in schizophrenia.

\section{Materials and Methods}

\section{Subjects}

Seventeen right-handed schizophrenia patients (age, 25-39 years; 13 males) and 17 right-handed healthy volunteers (control group; age, $28-36$ years; 12 males) participated in the first experiment. Three schizophrenia patients and one healthy subject were excluded due to excessive head motion $(>1 \mathrm{~mm})$ during the fMRI procedure in both experimental tasks. Some of the remaining subjects had severe head motions during the performance of one of the experimental tasks, and were therefore removed from the analysis of the task in which they moved but not from the analysis of the other task. In total, 14 healthy subjects and 10 schizophrenia patients participated in the $n$-back task (NBK), and nine healthy and eight schizophrenia patients participated in the verb generation task (VGM). Seven controls and four patients were included in the analysis of both tasks. The schizophrenia patients were inpatients at the Tirat Hacarmel Mental Health Center and were medicated with either haloperidol or other atypical antipsychotic drugs. One subject was also medicated with depalept, and another subject was medicated with anaphranil. The participants in the second experiment were seven first-episode schizophrenia patients (age, 19-29 years; four males) and six healthy controls (age, 25-34 years; all males). Two patients were excluded due to severe head motions $(>1 \mathrm{~mm})$. All participants were native Hebrew speakers. The schizophrenia patients of the second experiment were inpatients at the Beer Yaakov Mental Health Center and were medicated with perphenan, risperdal, or zyprexa. Eligibility for participation in the study was limited to right-handed volunteers between the ages of 19-39 years. A psychiatrist verified the patients' diagnoses according to the Structured Clinical Interview for DSM-IV Axis I, Patient Edition (First et al., 1994). Patients with any significant medical or neurological illness or who were pregnant or substance abusers were excluded from the study. Medical and neurological illnesses were ruled out on the basis of medical history, physical and neurological examinations, routine laboratory investigation, and medical records.

All study entrants provided written informed consent after receiving a full explanation on the nature of the study and potential risks and benefits to study participants. The study was approved by the Tirat Hacarmel Mental Health Center and the Tel Aviv Sourasky Medical Center Institutional Review Boards.

\section{fMRI paradigms}

Verb-generation task. While being scanned, eight schizophrenic patients (age, 30-38 years; six males) and nine healthy controls (age, 30-34 years; seven males) participated in an auditory language task or listened to periods of music, interspersed with periods of no stimuli. Before entering the fMRI phase of the investigation, all subjects underwent a preparatory session in which adequate task compliance was assured. The task was composed of 18 spoken Hebrew words presented through headphones during three 18 s periods. Each block consisted of six different words, at a rate of $1 / 3 \mathrm{~Hz}$. There were $24 \mathrm{~s}$ of no stimuli at the beginning and at the end of the paradigm. Although MRI scanning is inherently accompanied by noise, the noise is constant, therefore no stimuli can be considered as rest. Each verbal period was alternated with three $18 \mathrm{~s}$ periods of classical music and five $9 \mathrm{~s}$ periods of rest.

The words were nouns comprised of three to five letters and described commonly used objects, such as a brush, a table, etc. The subjects were instructed to covertly generate a verb that best described what they could do with the object that was named. Each verbal period was terminated with a tone. During the periods of music, participants were instructed to listen passively to the music and to do nothing during the rest condition. All subjects had performed with an $>90 \%$ accuracy score in a prior, overt response practice session on the task.

n-Back task. While being scanned, 10 schizophrenic patients (age, 28-39 years; six males) and 14 healthy controls (age, 29-36 years; nine males) participated in an $n$-back task composed of two conditions (zeroback and two-back), interspersed with periods of no stimuli. The visual stimuli consisted of 60 achromatic numbers with a red fixation point in the center of the image. The task lasted $3.39 \mathrm{~min}$, with six alternating blocks of the two conditions (three zero-back blocks and three two-back blocks) and eight baseline blocks. Conditions were presented following instructions as a series of one-digit numbers. During the zero-back condition, subjects were required to indicate when the number 9 appeared. During the two-back condition, subjects were required to indicate when a number had appeared two steps before. In the baseline condition, subjects were instructed to maintain fixation using a point in the middle of the screen. Each block consisted of 10 stimuli. Stimuli presentation rate was $2 \mathrm{~s}$ ( $1 \mathrm{~s}$ of a number interposed with a $1 \mathrm{~s}$ blank screen). There were $21 \mathrm{~s}$ of no stimuli at the beginning and $6 \mathrm{~s}$ at the end of the paradigm. Between blocks there was a $6 \mathrm{~s}$ period of rest. The stimuli were projected via an LCD projector (Epson MP 7200) onto a screen located on the head coil in front of the subject. Subjects viewed the screen through a tilted mirror. All subjects underwent a preparatory session in which adequate task compliance was assured.

Rest. While being scanned, five schizophrenia patients (age, 19-25 years; three males) and six healthy controls (age, 25-34 years; all males) were asked to close their eyes and not think of anything specific for $4.85 \mathrm{~min}$.

\section{Brain scanning: NBK and VGM tasks}

Imaging was performed on a GE 1.5T Signa Horizon LX 8.25 echo speed scanner with a resonant gradient echoplanar imaging system. All images were acquired using a standard quadrature head coil. The scanning session included anatomical and functional imaging. The anatomical imaging consisted of 27 contiguous axial T1-weighted slices of $4 \mathrm{~mm}$ thickness, with $1 \mathrm{~mm}$ gaps that were prescribed based on a sagittal localizer and covered the whole brain. In addition, a three-dimensional (3D) spoiled gradient echo sequence with high resolution (slice thickness, 2 $\mathrm{mm}$ ) was acquired for each subject. The functional imaging included $\mathrm{T} 2^{\star}$-weighted images that were acquired at the same locations as the spin-echo T1-weighted anatomical images, at runs of 1190 images each (70 images per slice). BOLD contrast was acquired with gradient-echo echo-planar imaging (EPI) sequence (TR, $3000 \mathrm{~ms}$; TE, $55 \mathrm{~ms}$; flip angle, $90^{\circ}$; FOV, $24 \times 24 \mathrm{~cm}^{2}$; matrix size, $\left.80 \times 80\right)$.

\section{Brain scanning: rest}

Brain scanning was done by a 3T MRI scanner (GE Signa EXCITE) with an eight-channels head coil. fMRI was performed with gradient EPI sequence of functional T2*-weighted images (TR, $3000 \mathrm{~ms}$; TE, $35 \mathrm{~ms}$; flip angle, $90^{\circ}$; FOV , $20 \times 20 \mathrm{~cm}$; matrix size, $\left.64 \times 64\right)$ divided into 38 axial slices ( $3 \mathrm{~mm}$ thick with no gap) covering the entire brain.

\section{Imaging data analysis}

fMRI data was processed using BrainVoyager QX 2.1 software package (Brain Innovation). The first six images of each functional scan were discarded. Preprocessing of functional scans included 3D motion correction, linear trend removal, slice scan time correction, and spatial smoothing using a Gaussian filter of $8 \mathrm{~mm}$ full width at half maximum value (FWHM) correction and temporal high-pass filtering with a cutoff frequency of $0.015 \mathrm{~Hz}$ for the VGM and NBK tasks and $0.01 \mathrm{~Hz}$ for the rest condition. Functional images were aligned with high-resolution anatomical volumes using trilinear interpolation, and the anatomical and functional images were transformed to the Talairach coordinate system. The cortical surface was reconstructed from the high-resolution anatomical images, separately for each subject; the procedure included segmenting the gray and white matter and inflating gray matter. Three-dimensional statistical parametric maps were calculated separately for each subject using a general linear model (GLM) in which all stimuli conditions were 
positive predictors with a hemodynamic lag of $6 \mathrm{~s}$. Motion parameters from head movements were used as predictors in first-level GLM.

For the rest runs, we removed non-neuronal contributions to the BOLD signal by regressing both motion parameters and the time course of the BOLD signal from ventricles for each subject.

Calculation of significance values in the activation map was based on the individual voxel significance and a correction for multiple comparisons, as follows. We calculated the probability of a false positive from the frequency count of cluster sizes within the entire cortical surface, using a Monte Carlo simulation (BrainVoyager QX 2.1 Cluster-Level Statistical Threshold Estimator plug-in). The computation of the minimum cluster threshold was accomplished via Monte Carlo simulation of the random process of image generation; a minimum cluster size threshold was automatically set, which yields $5 \%$ protection against false-positive detection at the cluster level. Finally, multi-subject maps were generated using random-effect analysis.

\section{Functional connectivity-subject correlation index}

For each subject's functional scan, the time course of each cortical voxel was transformed to units of percentage signal change, correlated with the time courses (TCs) of all other cortical voxels, and then averaged. Voxels with a mean signal intensity that was lower than 100 were discarded as noise, as regions outside the brain show similar values. The number of discarded voxels was similar for the patient and control groups for all experimental conditions. This procedure yielded a single correlation index per voxel, which represented the degree of correlation between the voxel and all other cortical voxels. The correlation indices (CI) of all cortical voxels were presented in a cortical map form and were averaged to produce a single global correlation value per subject, here referred to as the subject connectivity index (SCI). The SCI was computed separately for each subject in each experimental condition.

For each experimental condition, a two-sided $t$ test was used to determine whether the SCIs of the schizophrenic and control groups were statistically different from each other.

\section{Group-level analysis}

The average correlation index per voxel was computed separately for each group by averaging the correlation indices of each voxel between all the group's subjects. Coregistration of the subjects' voxels space was done in Talairach coordinate system on the $3 \mathrm{D}$ volume space (Talairach and Tournoux, 1988). The resulting averaged voxel correlations were then projected onto a representative individual's cortical surface.

Disparity indices were computed to measure the degree of difference between the two groups in terms of the average correlation index of each voxel. The gray matter cortical voxels of all subjects were intersected and the correlation coefficients were converted to $z$ values using Fisher's $r$-to- $z$ transformation to improve normality. The correlation indices of all voxels were then extracted and a two-tailed $t$ test was performed to determine significant voxel-by-voxel correlation differences across conditions between the two groups. The resulting $t$ values were projected onto a representative individual's cortical surface, forming functional connectivity disparity maps. These maps show voxels in which the healthy group had significantly higher functional connectivity than the schizophrenic group. A correction for multiple comparisons was performed by calculating the probability of a false positive from the frequency count of cluster sizes within the entire cortical surface, using a Monte Carlo simulation as noted above.

\section{Correlation between disparity index and task-related activity}

To examine the relation between the level of task-related activity in each voxel and the disparity index, we plotted the level of task-related activity ( $t$ score from GLM) and the disparity index score for all cortical gray matter voxels in both tasks. To test the significance of this correlation, we used a permutation test in which the scores for each voxel were randomly shuffled and correlated. This was repeated 10,000 times and the true correlation score was compared with the 95th percentile of the resulting distribution. A linear regression between the task activity and disparity index was also computed.

\section{Voxel activity and variance}

To ascertain that connectivity differences were not due to low response amplitudes in the schizophrenia patient group, we examined the relation between voxel activity and BOLD signal variance. The data were analyzed by comparing the fluctuations of the BOLD signal between groups and behavioral states. For each voxel in the cortical gray matter, we calculated the variance of the voxel's TC. We then conducted an ANOVA to check for Variance $\times$ Group (healthy, schizophrenia) $\times$ Task $(\mathrm{NBK}, \mathrm{VGM}$, rest) interactions.

\section{Distance-dependent analysis}

Another method for assessing the level of disparity between the two groups was to determine the difference in connectivity within various cluster sizes. The sizes of the tested clusters were $9 \mathrm{~mm}\left(3^{3}\right.$ voxels $), 15$ $\mathrm{mm}\left(5^{3}\right.$ voxels $), 30 \mathrm{~mm}$ ( $10^{3}$ voxels $)$, and $45 \mathrm{~mm}$ ( $15^{3}$ voxels $)$.

The TC of each voxel in a subject's brain was normalized and averaged with the normalized TCs of all neighboring voxels within a given cluster size in a distance-dependent manner, so that proximal voxels received higher weight compared with distal voxels. The voxel weights were assigned according to a Gaussian curve with FWHM of the given cluster size. The resulting averaged TC was correlated with the original voxel's TC to yield a single correlation value that represented the level of correlation between a single voxel and the surrounding voxels in a specific cluster size. For each voxel, the number of neighboring voxels per cluster size was calculated. If $50 \%$ or more of the neighboring voxels were missing (e.g., voxels at the very edge of the cortex), the voxel was discarded. A group $t$ test analysis was then conducted as described for the disparity index calculation.

\section{Results}

\section{Task-related differences in FC}

In the first experiment, 14 chronic schizophrenia patients and 16 healthy controls were scanned while performing a working memory NBK task and a VGM task. We computed a correlation index, which indicated the average FC level of each voxel with every other voxel in the cortex. A subject's CI was computed by determining the correlation each of his cortical voxels had with every other voxel in the cortex, and then averaging across these correlations (see Materials and Methods, above). We performed this analysis for each subject separately and then averaged across individuals of each group to generate a CI map for each group (Fig. 1).

Cortical connectivity was compared across the schizophrenia and control groups by generating a disparity index (DI) for each voxel. This comparison enabled us to identify cortical regions with significant FC differences between the groups. Cortical connectivity was significantly weaker in the schizophrenia group in multiple cortical areas in both the NBK (Fig. 2c) and VGM (Fig. $3 c)$ tasks. The regions showing the greatest DI for the NBK task included the superior frontal gyrus (SFG), central sulcus (CS), precuneus (PCUN), inferior parietal lobe, inferior temporal gyrus, and fusiform gyrus (FG). We wondered whether the appearance of high DI in these particular cortical areas was related to the pattern of cortical areas activated by the task. To examine this, we superimposed the disparity map shown in Figure $2 c$ and the NBK activation map of the schizophrenia and control groups shown in Figure $2 a$. The results are shown in Figure $2 b$ and reveal that the regions exhibiting significant DI were those that were not activated by the task. To test the complementary nature of DI and task activity more stringently, we relaxed the threshold of the activity maps $(p<$ 0.34 ) (Fig. $2 a$, right) and again found nearly no overlap between regions with high DI and regions activated by the task (Fig. $2 b$, right). Similar results were found for the right hemispheres.

A similar result was found in the VGM task, which showed a higher and more widespread DI. Here, regions of the premotor 


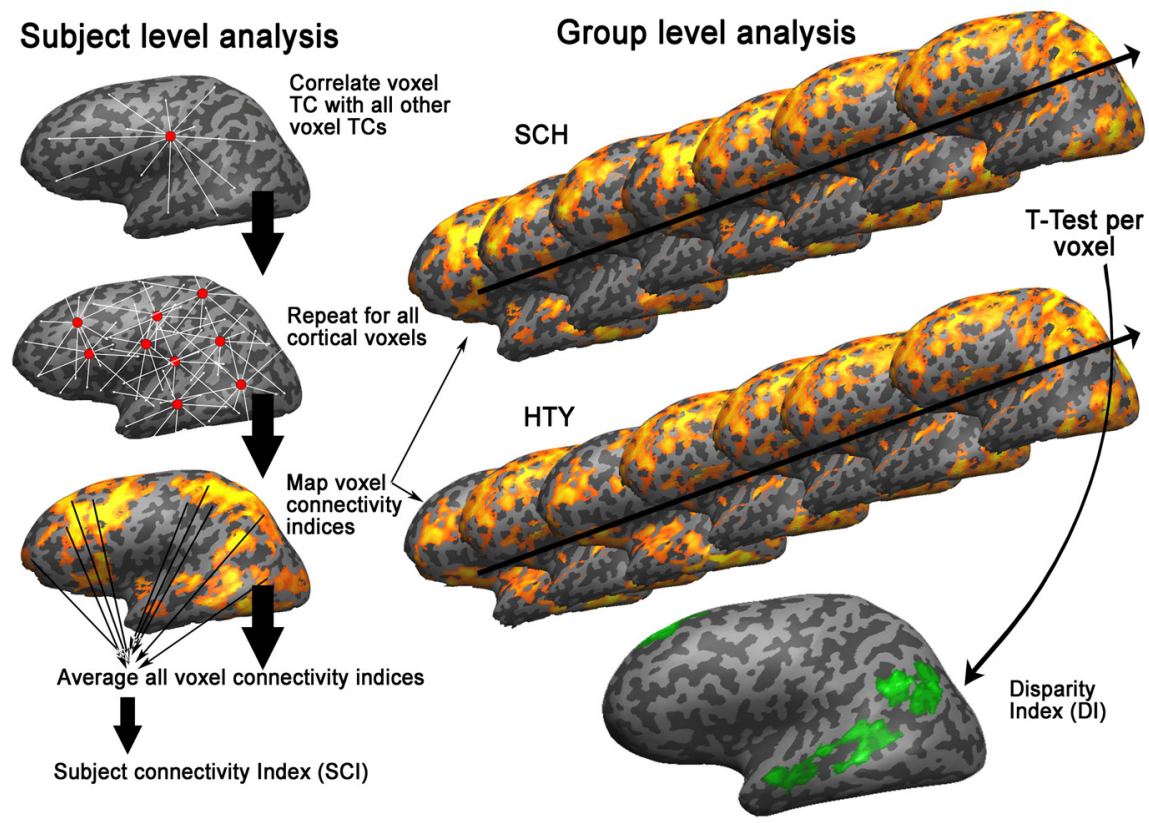

Figure 1. Schematic of data analysis pipeline. Left, Subject level analysis. For each subject's functional scan, each voxel's time course was correlated with the time course of all other voxels (top). This was repeated for all cortical gray matter voxels, so that each voxel had a single correlation index that was the mean of its correlation with all other voxels ( $\mathrm{Cl}$; middle). The voxel correlation index was projected onto spatial maps; the results from all voxels were averaged to produce the SCI (bottom). Right, Group level analysis. The voxel connectivity maps for all subjects in each group were subjected to a voxel-by-voxel between-group $t$ test. The resulting functional connectivity disparity maps show voxels in which one group had significantly higher functional connectivity. HTY, Healthy controls; SCH, schizophrenia patients.

cortex (PMC), CS, postcentral sulcus and gyrus, intraparietal sulcus (IPS), and anterior cingulate cortex exhibited high DI scores. Again, the regions showing high DI largely avoided the taskactivated regions. At the lower threshold, the picture was similar with little overlap between high DI regions and task-activated regions (Fig. 3). Interestingly, some regions, such as the PMC and IPS, which did not exhibit significant DI in the NBK task, exhibited significant DI in the VGM task. This difference in DI mirrors the difference in the responses of these areas during the two tasks (strong response during the NBK task and no response during the VGM task).

Thus, our results reveal an intriguing relationship between task-related cortical activation and DI, with DI localized to regions not activated or deactivated by the task. To further test this result, we compared the disparity index of each cortical voxel (i.e., to what extent the FC of the voxel differed between the control and schizophrenia groups) and the voxel's amplitude of response during the task (Fig. 4). An inverse correlation between DI and task activation was found $(r=-0.29)$, indicating that activated voxels exhibited lower DI scores. To test the significance of this correlation, we used a randomization test in which we shuffled the identities of the voxels' activation and DI values, and then performed a correlation analysis. This was repeated 10,000 times to get a distribution of random correlations expected by chance from the same dataset. The true negative correlation between activity and DI exceeded the 95th percentile of this distribution ( $p<0.0001$; Fig. 4$)$. A linear regression analysis between the variables was also conducted and found to be significant $(F=$ 3.68, $p=0.01$ ).

As can be seen, voxels that showed close to zero activation level (near 0 on the $x$-axis) manifested a large range of disparity values. However, as we move away from the 0 activity range, we see a significant trend for lower disparity values associated with higher taskactivation ones.

\section{Possible confounds}

While our results revealed a striking difference between the FC of schizophrenia patients and healthy controls, it should be noted that changes in the overall measured FC may be caused by two different brain phenomena. Two main possibilities could account for this. First, a real dissociation of coherent activity between different cortical sites-this is the general mechanism assumed to underlie changes in FC. However, an alternative mechanism that may also lead to a more confined FC is a lower amplitude of the local signal fluctuations. In the presence of noise, which corrupts these fluctuations to a substantial degree, lower amplitude fluctuations may be lost, which would lead to lower FC spread (Nir et al., 2008). To examine whether such changes in local signal amplitude may underlie the observed disparity in the present study, we examined whether the observed disparity was due to a difference in the variance (amplitude) of the spontaneous fluctuations exhibited by the two groups. We calculated an ANOVA comparing the variance of the BOLD signal fluctuations in the control and schizophrenia groups (see Materials and Methods, above). The results of this analysis showed that there was no significant difference in the variance between the groups or tasks, nor any significant interaction (all $F<1$ ). This result confirms that the differences in FC were not due to different amplitudes of BOLD response in the schizophrenia group.

\section{Task performance}

Another possible confounding variable is the task performance. If individuals from the schizophrenia group performed the task differently than controls, differences in connectivity could be related to differences in behavior. Schizophrenia patients indeed performed the NBK task less accurately than controls (schizophrenia group, mean $=94.5, \mathrm{SD}=3.14$; control group: mean $=$ 97.96, $\mathrm{SD}=2 ; p<0.05)$. There was, however, no correlation between the average CI measure and task performance $(r=0.22$, $p=0.4$ ) or in either of the groups (healthy group: $r=-0.13, p=$ 0.76 ; schizophrenia group: $r=0.41, p=0.35$ ).

In the VGM task, no overt performance was required, yet schizophrenia patients showed their ability to perform this task in a preceding training session with overt responses.

\section{Stimuli-correlated movement}

As stimuli-correlated head movements may bias activity maps, we correlated each subject's six motion parameters (three translations and three rotation axes) with the task design matrix. Correlation coefficients were converted to $z$ values using Fisher's $r$-to- $z$ transformation to improve normality. We then used a onesample $t$ test to determine whether the correlation coefficients were significantly different from zero. Furthermore, we compared the stimuli-correlated movement (SCM) between the 

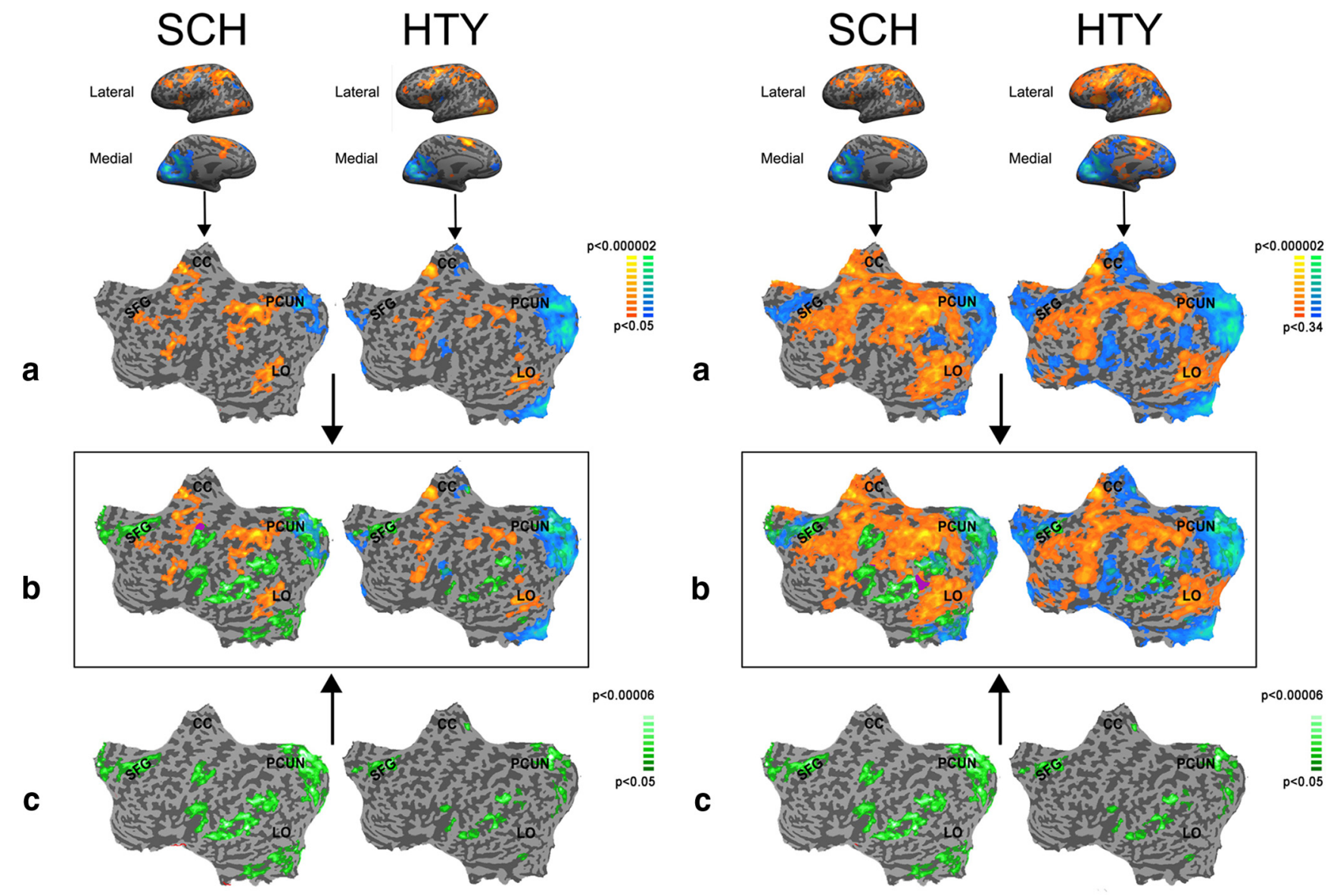

Figure 2. Voxel task related-activity and schizophrenia index maps: NBK task. $\boldsymbol{a}$, Group activity maps (random-effects analysis corrected for multiple comparisons) for the NBK task. Healthy (HTY) and schizophrenia $(\mathrm{SCH})$ group results shown on inflated and flattened maps. Results shown for left hemisphere; right hemisphere showed similar results. $c$, DI map (map of voxels showing significant difference between the schizophrenia and control groups in the level of connectivity; see Materials and Methods) shown on flattened cortical maps. $\boldsymbol{b}, 0$ verlap between the two maps. Note voxels showing significant difference in the disparity index (control $>$ schizophrenia) are located in regions not activated by the task or showing task-negative activity (blue). Overlap between task-active voxels and regions of high Dl are shown in purple. Left, Activity effects at significant $p$ level. Right, Same effects as on the left, but with the activity map $p$ value relaxed ( $p<0.34)$. Note, even when $p$ value is lowered considerably, there is little overlap between task-activated voxels and disparity index voxels. This indicates that the effect of differences between the control and schizophrenia group is generally restricted to regions not activated by the task. CC, Cingulate cortex; LO, lateral occipital cortex.

healthy and patient groups using a two-sided $t$ test to look for possible group differences. The results showed that SCM was not significantly different from zero in any of the groups or tasks (all ps $>0.7)$, nor was there any difference between the groups in the extent of the SCM (all ps > 0.75).

\section{Illness duration}

To test for effects of illness duration (which is typically correlated to antipsychotic exposure) as a contributing factor to the FC deficits, we correlated the illness duration with the subjects' CI. This analysis revealed no correlation between the CI for the $n$-back task $(r=0.27, p=0.44)$ or the VGM task $(r=$ $-0.14, p=0.71)$.

\section{FC during rest}

The first two experiments revealed an intriguing complementary relationship between cortical regions that were activated by the task and the FC disparity between schizophrenia and control groups. Given this observation, it may be expected that during rest (lack of explicit task), the FC disparity between schizophrenia and controls should reach its maximum. To examine this assumption, we ran a second experiment using rsfMRI. Five first- episode schizophrenia patients and six healthy controls were scanned while resting with eyes closed.

The second experiment was performed with a different cohort of subjects, using a different fMRI magnet. Schizophrenia patients were first-episode rather than chronic schizophrenia patients and hence were not chronically medicated. Despite these differences, we again found significantly decreased CI in the schizophrenia group during full rest compared with the control group (Fig. 5). When comparing the connectivity in the three experiments, a gradual increase in the strength and spread of the DI was apparent when moving from the NBK task, through the VGM task, and finally the rest condition. These results were also evident when we averaged the disparity index across all voxels, deriving an averaged DI value for the entire cortex (see Materials and Methods, above; and Fig. 1) as indicated by the bar insets above each map.

To gain some insight into the inverse relationship between task-related activation and the FC disparity, we examined the FC (group CI) of the schizophrenia group and the controls during the three conditions: NBK, VGM, and rest. As can be seen, there was a striking difference between the FC of the control group (Fig. 5, top) and that of schizophrenia group (Fig. 5, third row) in 

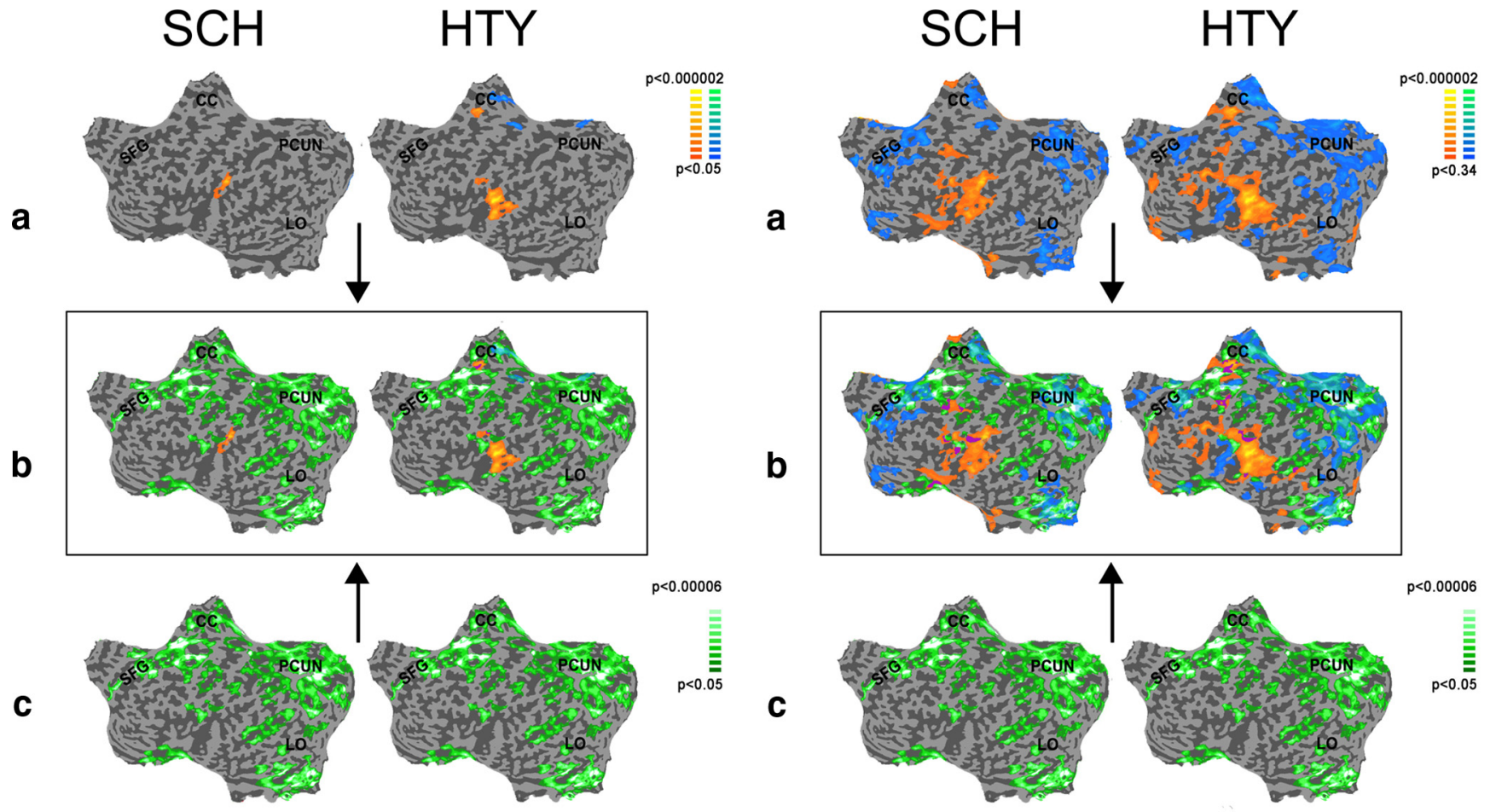

Figure 3. Voxel task-related activity and schizophrenia index maps: VGM task. $\boldsymbol{a}$, Group activity maps (random-effects analysis corrected for multiple comparisons) for the VGM task. Healthy (HTY) and schizophrenia (SCH) group results shown on inflated and flattened maps. Results shown for left hemisphere. c, Dl map (map of voxels showing significant difference between the schizophrenia and control groups in the level of connectivity; see Materials and Methods) shown on flattened cortical maps. $\boldsymbol{b}$, Overlap between the two maps. Note voxels showing significant difference in the disparity index (control $>$ schizophrenia) are located in regions not activated by the task or showing task negative activity (blue). Overlap between task active voxels and regions of high Dl are shown in purple. Left, Activity effects at significant $p$ level. Right, Same effects but with the activity map $p$ value relaxed $(p<0.34)$. Note, even when the $p$ value is lowered considerably, there is little overlap between task-activated voxels and disparity index voxels. This indicates that the effect of differences between the control and schizophrenia group is generally restricted to regions not activated by the task. CC, cingulate cortex; LO, lateral occipital cortex.

\section{Voxel task related activity and disparity index}

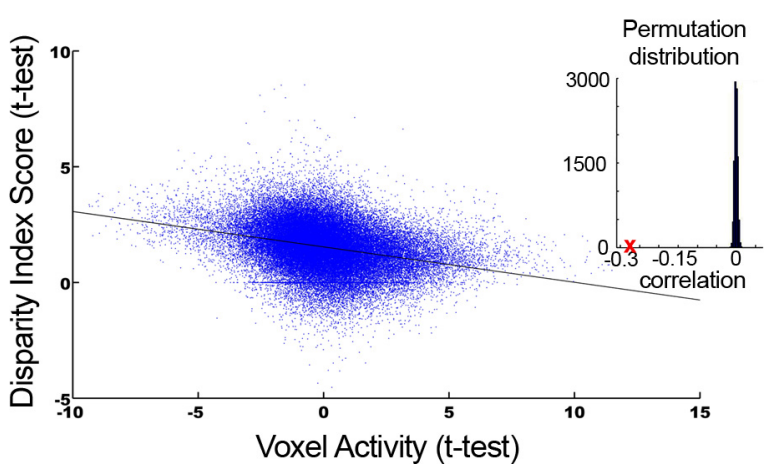

Figure 4. Correlation between disparity index and task-related activity. Scatter plot of voxel task-related activity (both tasks) and disparity index (both groups). The plot shows a clear and significant negative correlation $(r=-0.29)$, indicating an inverse relationship between the level of task-related activity and the disparity index. The linear regression between the two is also significant $(F=3.68, p=0.01)$. Inset, Results of permutation test for correlation significance. True correlation value is much lower than the distribution created from 10,000 random permutations of the plot values shown in Figure $4(p<0.000)$.

each task. The difference between the two groups' averages was largest in the rest condition ( $t$ test, $p<0.00001$ ), smaller in the VGM task ( $t$ test, $p=0.02$ ), and smallest in the NBK task ( $t$ test, $p=0.12$, not significant). Although the rest experiment was conducted in a different MR scanner and on a different cohort of subjects, the results suggest that the CI in the schizophrenia group remained fairly constant between tasks. In contrast, the CI of the healthy group seemed to increase considerably between tasks. While the NBK task did not show a significant global difference in the average DI scores $(p=0.12)$, many regions (e.g., PCUN, SFG, inferior temporal gyrus) showed significant connectivity differences between the two groups, with controls showing higher connectivity than schizophrenia patients.

\section{Global versus local connectivity}

The CI represented a global average connectivity measure for each voxel with every other voxel in the cortex. We also computed a more local connectivity measure for each voxel by averaging the correlations of neighboring voxels at different cortical distances (see Materials and Methods, above). Specifically, we compared the correlation indices for several local neighborhood sizes (Fig. 6 ). This analysis revealed that the trend of lower CI in the schizophrenia group was evident for all neighborhood sizes ranging from 9 to $45 \mathrm{~mm}$ in diameters in both tasks as well as in the rest experiment. The differences between groups were significant for all cluster sizes in the VGM condition, and in all cluster sizes except the smallest one in the rest conditions. These findings indicate that reduced connectivity in the schizophrenia group is present at different spatial scales.

\section{Functional connectivity as a diagnostic measure}

The average CI across all cortical voxels (SCI) enabled accurate identification of all schizophrenia patients in the rest experiment (Fig. 7). All healthy control subjects showed higher FC than the schizophrenia subjects, suggesting it may be useful as an MRbased diagnostic criterion. This was also evident, to a lesser extent, in the VGM task at the small cluster size (Fig. 8). 


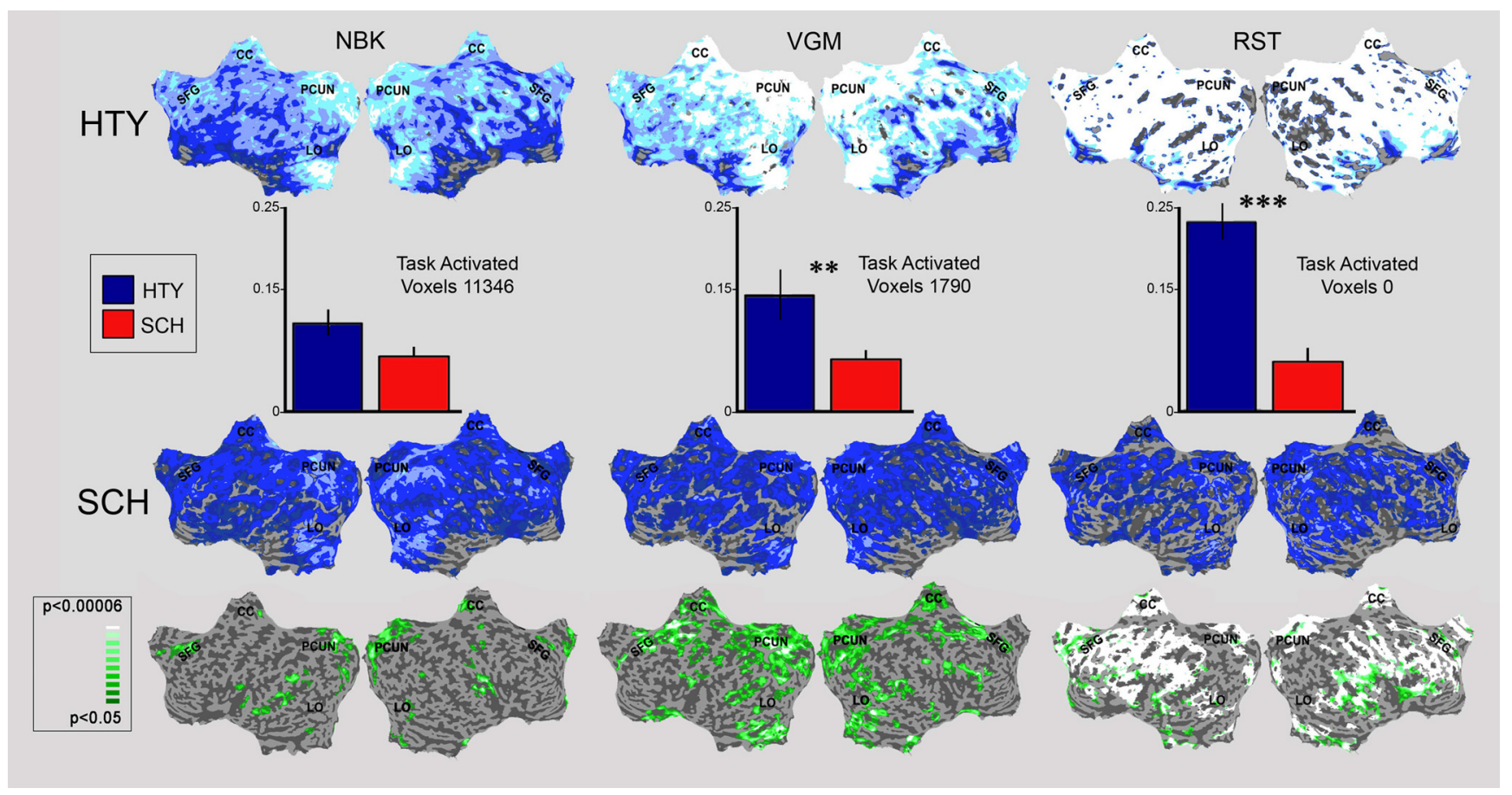

Figure 5. Group connectivity maps and disparity index per task. Top, Maps of connectivity index, showing voxels with high connectivity in healthy control group. Bar graphs show the average subject connectivity indices per group. Middle, Maps of connectivity index, showing voxels with high connectivity in the schizophrenia patient group. Bottom, Disparity index maps. Green-to-white colors indicate voxels in which healthy control subjects showed significantly higher correlation than the schizophrenia patient group. Note: no voxels showed higher correlation for the schizophrenia group and the effect becomes stronger in the VGM and rest runs in which fewer voxels were activated by the task. Error bars indicate SEM. CC, Cingulate cortex; L0, lateral occipital cortex; SCH, schizophrenia group; HTY, healthy controls. ${ }^{* *} p<0.02,{ }^{* * *} p<0.00001$.

Since the SCI is an averaged global measure, it could be argued that the reduced SCI in schizophrenia was a byproduct of canceling out positive and negative correlation values due to averaging. To examine this possibility, we computed the number of voxels showing negative correlation values. These voxels constitute $<10 \%$ of all voxels, with an average magnitude of -0.03 ; the average magnitude of the positive voxels was 0.12 . We then repeated the analysis with the negative voxels removed, and the results remained nearly identical. Comparison of the $p$ values of the first analysis $\left(p_{1}\right)$ to those of the analysis without negative voxels $\left(p_{2}\right)$ revealed negligible difference in the NBK task $\left(p_{1}=0.0264, p_{2}=0.0246\right)$, in the VGM task $\left(p_{1}=0.0177, p_{2}=0.0178\right)$, and in the rest experiment $\left(p_{1}=1.161 \times 10^{-4}, p_{2}=\right.$ $\left.1.117 \times 10^{-4}\right)$.

\section{Discussion}

Our results reveal a robust difference in functional connectivity patterns between healthy controls and schizophrenia patients. However, this difference was dependent on the activity state of the cortex and showed a striking complementary anatomical distribution to the task-activated regions (Fig. 2). Thus, areas showing high DI included the regions of the DMN that are consistently deactivated by extrinsically oriented tasks (Salomon et al., 2009; Preminger et al., 2011) as well as regions of the sensorymotor cortex that were specifically inactive or deactivated by the

\section{Clustersize: $3^{3}$ Clustersize: $5^{3} \quad$ Clustersize: $10^{3}$ Clustersize: $15^{3}$}
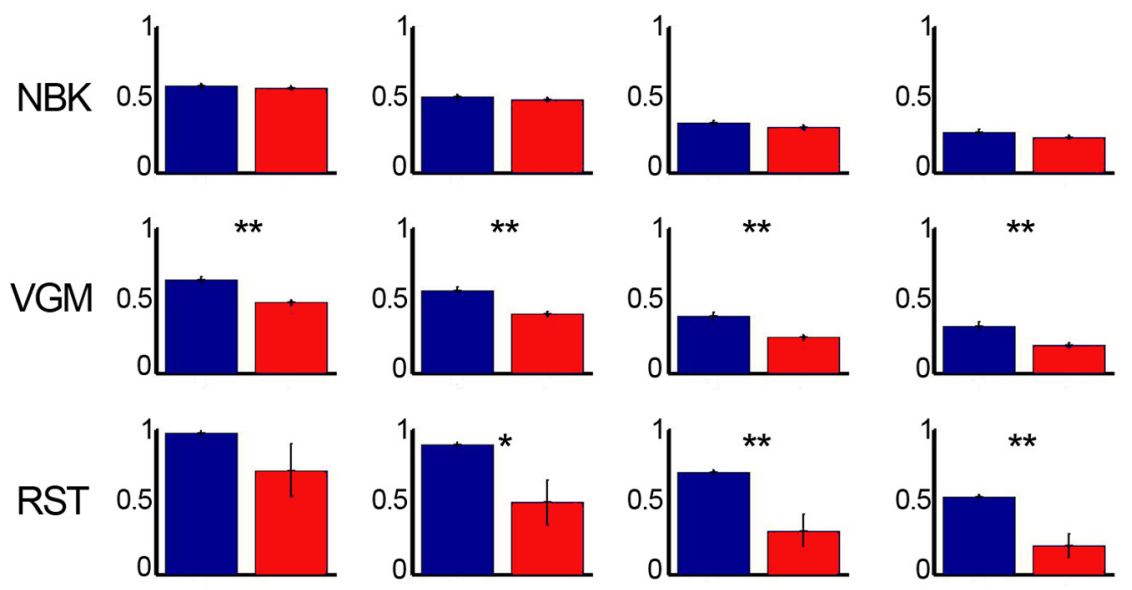

- HTY

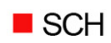

$* p<0.05 \quad * * p<0.005$

Figure 6. Connectivity averages for different cluster sizes per task. Top, NBK task. Middle, VGM task. Bottom, Rest (RST) condition. Note that differences for VGM and rest tasks are significant in nearly all (seven of eight) cluster sizes, indicating that the connectivity deficit is present at the local as well as global level. Error bars indicate SEM. SCH, Schizophrenia group; HTY, healthy controls.

tasks. Additionally, some regions showing significant DI differed between the two tasks, possibly due to the differences in task activity. This complementary nature of the DI and activity maps (Figs. 2, 3) suggests that the FC differences between the control and schizophrenia groups were modulated by cortical activity.

As expected from the complementarity between task activation and schizophrenia deficit, we found an inverse relationship between the response amplitude to a task and the DI between 


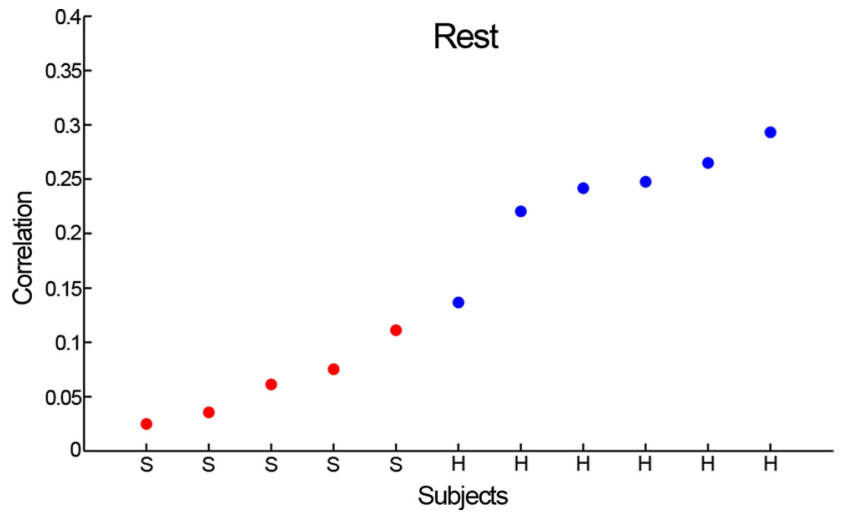

Figure 7. Subject connectivity indices per subject in rest task. Plot showing SCI. Note, subject connectivity index shows clear differentiation between schizophrenic patients (red) and healthy controls (blue) on a subject-by-subject level. The healthy controls showed higher connectivity levels.
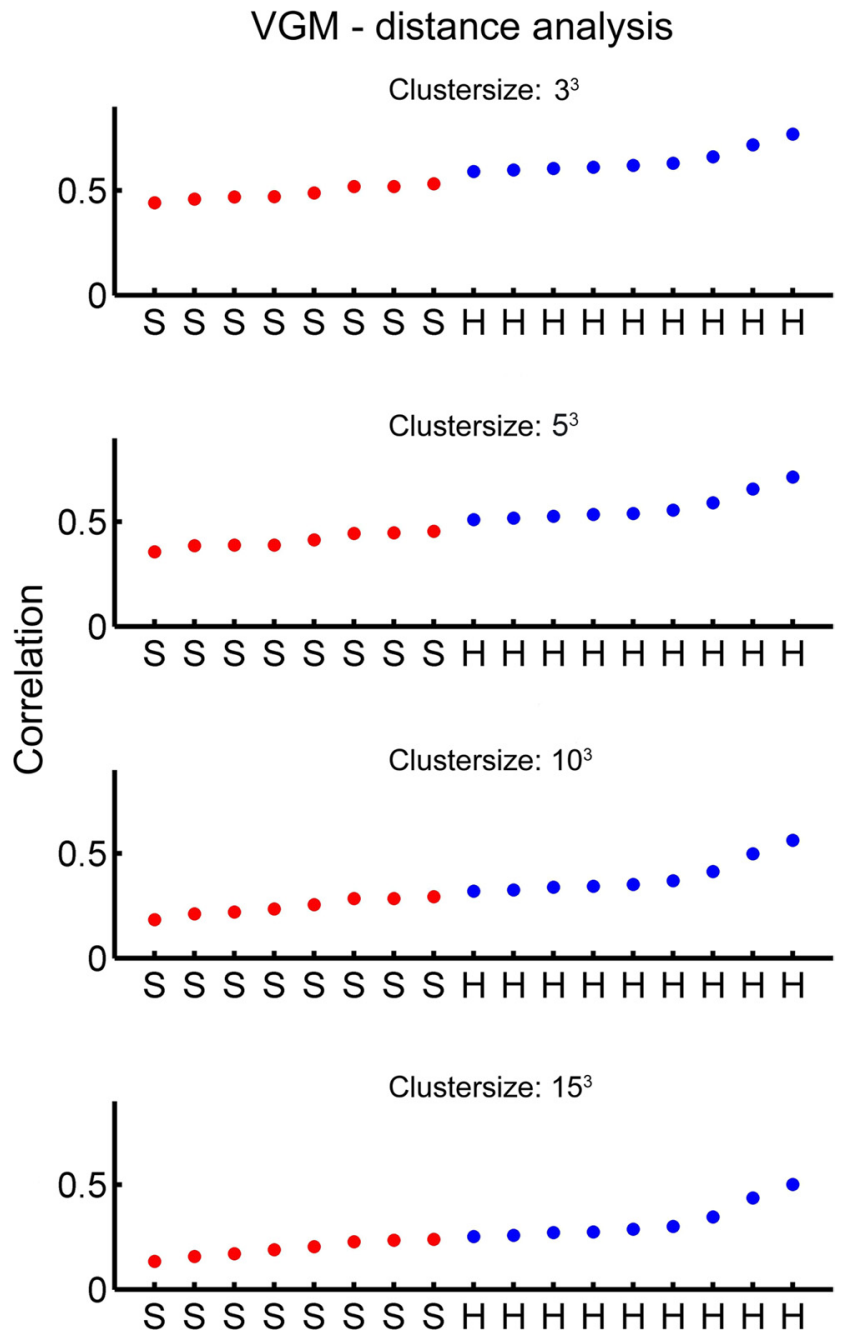

Subjects

Figure 8. Subject connectivity indices for different cluster sizes: VGM task. SCI for all subjects using different cluster sizes for the VGM task. Note that the schizophrenia group subjects ( $S$; red) consistently have lower correlation indices than the healthy group $(\mathrm{H}$; blue) and are clearly separable at the small cluster size. This highlights the promise of the SCl as a data-driven clinical index for schizophrenia. groups (Fig. 4). DI was particularly large in voxels that did not respond to the task. Note that voxels whose signal was corrupted (for example, due to MR susceptibility artifacts) would be expected to show no response and no DI. Such noisy voxels would reduce the described inverse correlation and may account for the large number of voxels exhibiting no response and no DI (Fig. 4). Thus, the differences in FC during tasks are derived primarily from task-deactivated or nonactivated voxels. Of these, the taskdeactivated voxels showed the highest levels of DI.

A straightforward prediction of this observation is that during a full rest condition, the DI would be larger. This prediction was confirmed by the rest experiment where the difference between the two groups was indeed much larger than in the task experiments (Fig. 5, histogram). During the rest experiment, this effect was sufficiently large to allow a case-by-case differentiation of schizophrenia patients from controls using a single data-driven index (Fig. 7). Thus, we found a striking reduction of connectivity in schizophrenic patients at rest compared with normal controls. Note that the patients participating in the rest experiment were first-episode patients, arguing against the attribution of the FC deficit to chronic medication.

At this stage, we can only speculate about the source of the statedependent difference between the schizophrenia and control groups. One possibility is that the reduced functional connectivity in schizophrenia is hard wired, e.g., may be related to reduced anatomical connectivity, as suggested previously (Schlösser et al., 2007; Camchong et al., 2011; Skudlarski et al., 2010). However, the reciprocal relationship between the structural and functional connectivity is unclear (Skudlarski et al., 2010) and abnormal structural connectivity would not explain the dynamic changes in FC as a function of cortical activity state. An alternate possibility is that the reduced functional connectivity in schizophrenia is related to abnormalities in the behavioral shift from a confined task-related connectivity state (Nir et al., 2006) to an idle, resting mode that is normally characterized by widespread FC.

Regardless of the exact source of the reduced connectivity in schizophrenia, this consistent reduction in functional connectivity could account for the observed task-related pattern of DI distribution. Thus, it should be noted that in healthy controls, unlike in schizophrenic patients, the FC shows a clear task-related anatomical focusing (also termed decoherence) (Nir et al., 2006, 2008; Schölvinck et al., 2010). If the patients group failed to show such task-driven focusing, this discrepancy could explain the enhanced DI in the inactive and, even more so, deactivated voxels. Thus, task-driven focusing could bring the control FC spread closer to that of schizophrenia patients, leading to less of a disparity between the healthy and schizophrenic groups in the taskactivated voxels and, conversely, to more enhanced DI in the inactivated voxels. Further research using paradigms leading to a range of activations and deactivation patterns is needed to substantiate this conjecture.

If the latter hypothesis is correct, then one would expect the schizophrenic brain to lack other resting state characteristics such as increased EEG alpha power (Adrian and Yamagiwa, 1935). Patients with schizophrenia indeed exhibit diminished alpha power during rest (Merrin and Floyd, 1992; Sponheim et al., 2000). Importantly, several studies have shown that this effect cannot be attributed to medication (Omori et al., 1995; Merrin and Floyd, 1996). A recent study has shown that treating alpha band deficiency by repetitive transcranial stimulation in the alpha frequency has significant therapeutic effects in schizophrenia patients (Jin et al., 2006). Finally, it has been reported that schizo- 
phrenic patients show a marked reduction in task-related activity, also known as task-related hypo-frontality (Manoach, 2003; Hill et al., 2004), as well as reduced deactivation in default mode network regions (Pomarol-Clotet et al., 2008). These findings could be explained by a deficit in schizophrenia patients' ability to go into a state of high connectivity during rest epochs, hence showing reduced activity compared with the continuously active baseline state.

It may be argued that the differences in FC are due to medication effects. Yet several lines of evidence argue against this option. First, the most robust abnormality in our study was obtained in first-episode patients who were not chronically medicated. This indicates that the reduced FC effect could not be due to long-term medication effects. Additionally, it has been shown that antipsychotic medications have a normalizing effect on the brain function of schizophrenic patients (Davis et al., 2005). Finally, previous studies have found altered connectivity in unmedicated patients in fMRI (Meyer-Lindenberg et al., 2005) and EEG (Omori et al., 1995) studies.

As discussed in the Introduction, the literature portrays a mixed view as to the FC differences between healthy individuals and schizophrenia patients. The present study may assist in clarifying these contradictory findings by illustrating that the FC disparity between schizophrenia patients and healthy controls is a variable that is strongly modulated by task activations. For example, in a continuous performance task, regions in which the taskrelated activity was reduced in the schizophrenia group showed reduced FC as well. Other regions in which the patient group showed increased task-related activity also showed increased levels of FC (Honey et al., 2005).

During rest, some studies, typically using a ROI-based approach, showed increased FC between some cortical regions (Zhou et al., 2007; Liu et al., 2010). Yet, similar to the present study, voxel-based studies and use of unrestricted methods have revealed significant reductions in FC during rest in schizophrenia patients (Liang et al., 2006; Camchong et al., 2011; Lynall et al., 2010).

Our study demonstrated that healthy individuals show a reduction in functional connectivity during cognitive tasks. This supports and confirms findings from previous studies showing that FC between different category-specific visual regions is confined and reduced during a visual categorization task. Such FC is higher when compared with rest in darkness (Nir et al., 2006) and stronger during rest than during activity or arousal (Cordes et al., 2000; Schölvinck et al., 2010). A direct prediction of the present study is that the spread of functional connectivity will be strongly modulated when switching between task blocks and no-task epochs.

Our study used a data-driven analysis that shows promise as a diagnostic tool in schizophrenia. We report a successful case-bycase separation between schizophrenia patients and healthy individuals using a data-driven brain measure. Further studies of global FC measures in schizophrenia patients and other psychiatric groups are needed to assess the specificity of these phenomena. The robust nature of the FC abnormality has important implications for better diagnosis and understanding of schizophrenia. The complementary nature of task activity and FC differences shown here provides an important insight into the mechanism underlying FC abnormalities in schizophrenia and may explain the contradictory findings found in the study of FC in schizophrenia patients.

\section{References}

Adrian E, Yamagiwa K (1935) The origin of the Berger rhythm. Brain $58: 323-351$

Bhugra D (2005) The global prevalence of schizophrenia. PLoS Med 2:e151. Bleich-Cohen M, Strous RD, Even R, Rotshtein P, Yovel G, Iancu I, Olmer A, Hendler T (2009) Diminished neural sensitivity to irregular facial expression in first-episode schizophrenia. Hum Brain Mapp 30:2606-2616.

Bluhm R, Miller J, Lanius RA, Osuch EA, Boksman K, Neufeld RW, Théberge J, Schaefer B, Williamson P (2007) Spontaneous low-frequency fluctuations in the BOLD signal in schizophrenic patients: anomalies in the default network. Schizophr Bull 33:1004-1012.

Camchong J, MacDonald AW 3rd, Bell C, Mueller BA, Lim KO (2011) Altered functional and anatomical connectivity in schizophrenia. Schizophr Bull: 37:640-650.

Cordes D, Haughton VM, Arfanakis K, Wendt GJ, Turski PA, Moritz CH, Quigley MA, Meyerand ME (2000) Mapping functionally related regions of brain with functional connectivity MR imaging. Am J Neuroradiology 21:1636-1644.

Davis CE, Jeste DV, Eyler LT (2005) Review of longitudinal functional neuroimaging studies of drug treatments in patients with schizophrenia. Schizophr Res 78:45-60.

First MB, Spitzer RL, Gibbon M, Williams JB (1994) Structured Clinical Interview for DSM-IV Axis I Disorders, Patient Edition (SCID-P), version 2. New York: American Psychiatric.

Friston KJ (1998) The disconnection hypothesis. Schizophr Res 30: $115-125$.

Friston KJ, Frith CD (1995) Schizophrenia: a disconnection syndrome. Clin Neurosci 3:89-97.

Friston KJ, Frith CD, Liddle PF, Frackowiak RS (1993) Functional connectivity: the principal-component analysis of large (PET) data sets. J Cereb Blood Flow Metab 13:5-14.

Garrity AG, Pearlson GD, McKiernan K, Lloyd D, Kiehl KA, Calhoun VD (2007) Aberrant "default mode" functional connectivity in schizophrenia. Am J Psychiatry 164:450-457.

Hill K, Mann L, Laws KR, Stephenson CM, Nimmo-Smith I, McKenna PJ (2004) Hypofrontality in schizophrenia: a meta-analysis of functional imaging studies. Acta Psychiatr Scand 110:243-256.

Honey GD, Pomarol-Clotet E, Corlett PR, Honey RA, McKenna PJ, Bullmore ET, Fletcher PC (2005) Functional dysconnectivity in schizophrenia associated with attentional modulation of motor function. Brain 128:2597-2611.

Jafri MJ, Pearlson GD, Stevens M, Calhoun VD (2008) A method for functional network connectivity among spatially independent resting-state components in schizophrenia. Neuroimage 39:1666-1681.

Jin Y, Potkin SG, Kemp AS, Huerta ST, Alva G, Thai TM, Carreon D, Bunney WE Jr (2006) Therapeutic effects of individualized alpha frequency transcranial magnetic stimulation (\{alpha\} TMS) on the negative symptoms of schizophrenia. Schizophrenia bulletin 32:556-561.

Lawrie SM, Buechel C, Whalley HC, Frith CD, Friston KJ, Johnstone EC (2002) Reduced frontotemporal functional connectivity in schizophrenia associated with auditory hallucinations. Biol Psychiatry 51:1008-1011.

Liang M, Zhou Y, Jiang T, Liu Z, Tian L, Liu H, Hao Y (2006) Widespread functional disconnectivity in schizophrenia with resting-state functional magnetic resonance imaging. Neuroreport 17:209-213.

Liu H, Kaneko Y, Ouyang X, Li L, Hao Y, Chen EYH, Jiang T, Zhou Y, Liu Z (2010) Schizophrenic patients and their unaffected siblings share increased resting-state connectivity in the task-negative network but not its anticorrelated task-positive network. Schizophr Bull. Advance online publication. Retrieved August, 2010. doi:10.1093/schbul/sbq074.

Liu Y, Liang M, Zhou Y, He Y, Hao Y, Song M, Yu C, Liu H, Liu Z, Jiang T (2008) Disrupted small-world networks in schizophrenia. Brain 131:945-961.

Lynall ME, Bassett DS, Kerwin R, McKenna PJ, Kitzbichler M, Muller U, Bullmore E (2010) Functional connectivity and brain networks in schizophrenia. J Neurosci 30:9477-9487.

Manoach DS (2003) Prefrontal cortex dysfunction during working memory performance in schizophrenia: reconciling discrepant findings. Schizophr Res 60:285-298.

McGuire PK, Frith CD (1996) Disordered functional connectivity in schizophrenia. Psychological medicine 26:663-667.

Merrin EL, Floyd TC (1992) Negative symptoms and EEG alpha activity in schizophrenic patients. Schizophr Res 8:11-20. 
Merrin EL, Floyd TC (1996) Negative symptoms and EEG alpha in schizophrenia: a replication ${ }^{*}$ 1. Schizophr Res 19:151-161.

Meyer-Lindenberg A, Poline JB, Kohn PD, Holt JL, Egan MF, Weinberger DR, Berman KF (2001) Evidence for abnormal cortical functional connectivity during working memory in schizophrenia. Am J Psychiatry 158:1809-1817.

Meyer-Lindenberg AS, Olsen RK, Kohn PD, Brown T, Egan MF, Weinberger DR, Berman KF (2005) Regionally specific disturbance of dorsolateral prefrontal-hippocampal functional connectivity in schizophrenia. Arch Gen Psychiatry 62:379-386.

Nir Y, Hasson U, Levy I, Yeshurun Y, Malach R (2006) Widespread functional connectivity and fMRI fluctuations in human visual cortex in the absence of visual stimulation. Neuroimage 30:1313-1324.

Nir Y, Mukamel R, Dinstein I, Privman E, Harel M, Fisch L, Gelbard-Sagiv H, Kipervasser S, Andelman F, Neufeld MY, Kramer U, Arieli A, Fried I, Malach R (2008) Interhemispheric correlations of slow spontaneous neuronal fluctuations revealed in human sensory cortex. Nat Neurosci 11:1100-1108.

Omori M, Koshino Y, Murata T, Murata I, Nishio M, Sakamoto K, Horie T, Isaki K (1995) Quantitative EEG in never-treated schizophrenic patients. Biol Psychiatry 38:303-309.

Pomarol-Clotet E, Salvador R, Sarró S, Gomar J, Vila F, Martínez A, Guerrero A, Ortiz-Gil J, Sans-Sansa B, Capdevila A, Cebamanos JM, McKenna PJ (2008) Failure to deactivate in the prefrontal cortex in schizophrenia: dysfunction of the default mode network? Psychol Med 38:1185-1193.

Preminger S, Harmelech T, Malach R (2011) Stimulus-free thoughts induce differential activation in the human default network. Neuroimage 54:1692-1702.

Salomon R, Malach R, Lamy D (2009) Involvement of the intrinsic/default system in movement-related self recognition. PLoS One 4:e7527.
Salvador R, Sarró S, Gomar JJ, Ortiz-Gil J, Vila F, Capdevila A, Bullmore E, McKenna PJ, Pomarol-Clotet E (2010) Overall brain connectivity maps show cortico-subcortical abnormalities in schizophrenia. Hum Brain Mapp 31:2003-2014.

Schlösser RG, Nenadic I, Wagner G, Güllmar D, von Consbruch K, Köhler S, Schultz CC, Koch K, Fitzek C, Matthews PM, Reichenbach JR, Sauer H (2007) White matter abnormalities and brain activation in schizophrenia: a combined DTI and fMRI study. Schizophr Res 89:1-11.

Schölvinck ML, Maier A, Ye FQ, Duyn JH, Leopold DA (2010) Neural basis of global resting-state fMRI activity. Proc Natl Acad Sci U S A 107:10238-10243.

Skudlarski P, Jagannathan K, Anderson K, Stevens MC, Calhoun VD, Skudlarska BA, Pearlson G (2010) Brain connectivity is not only lower but different in schizophrenia: a combined anatomical and functional approach. Biol Psychiatry 68:61-69.

Sponheim SR, Clementz BA, Iacono WG, Beiser M (2000) Clinical and biological concomitants of resting state EEG power abnormalities in schizophrenia. Biol Psychiatry 48:1088-1097.

Talairach J, Tournoux P (1988) Co-planar stereotaxic atlas of the human brain: 3-dimensional proportional system: an approach to cerebral imaging. New York: Thieme.

Whitfield-Gabrieli S, Thermenos HW, Milanovic S, Tsuang MT, Faraone SV, McCarley RW, Shenton ME, Green AI, Nieto-Castanon A, LaViolette P, Wojcik J, Gabrieli JD, Seidman LJ (2009) Hyperactivity and hyperconnectivity of the default network in schizophrenia and in first-degree relatives of persons with schizophrenia. Proc Natl Acad Sci U S A 106:1279-1284.

Zhou Y, Liang M, Tian L, Wang K, Hao Y, Liu H, Liu Z, Jiang T (2007) Functional disintegration in paranoid schizophrenia using resting-state fMRI. Schizophr Res 97:194-205. 